PROCEEDINGS OF THE

AMERICAN MATHEMATICAL SOCIETY

Volume 135, Number 6, June 2007, Pages 1843-1849

S 0002-9939(07)08747-3

Article electronically published on January 5, 2007

\title{
EXISTENCE OF STRONG TRAVELLING WAVE PROFILES TO $2 \times 2$ SYSTEMS OF VISCOUS CONSERVATION LAWS
}

\author{
TONG YANG, MEI ZHANG, AND CHANGJIANG ZHU \\ (Communicated by Walter Craig)
}

\begin{abstract}
In this paper, we prove the existence of strong travelling wave profiles for a class of $2 \times 2$ viscous conservation laws when the corresponding invisid systems are hyperbolic. Besides some technical assumptions, the only main assumption is the hyperbolicity. Therefore, the existence theory can be applied to systems which are not strictly hyperbolic. Moreover, the characteristic fields can be neither genuinely nonlinear nor linearly degenerate.
\end{abstract}

\section{INTRODUCTION}

Consider a $2 \times 2$ system of hyperbolic conservation laws with artificial viscosity

$$
\left\{\begin{array}{l}
u_{t}+f(u, v)_{x}=\varepsilon u_{x x}, \\
v_{t}+g(u, v)_{x}=\varepsilon v_{x x},
\end{array}\right.
$$

where $\varepsilon>0$ is a constant. In what follows, we assume that $f \in C^{2 m+1}\left(\mathbb{R}^{2}\right)$ and $g \in C^{2 n+1}\left(\mathbb{R}^{2}\right)$, where $m$ and $n$ are any non-negative integers. A lot of work has been done on the existence of solutions to the $2 \times 2$ systems of conservation laws with two constant end states; cf. [6, 7, 8, 9, 10, and the references therein. In this paper, we assume that the flux functions $f(u, v)$ and $g(u, v)$ satisfy the following conditions:

(H1) $\quad \frac{\partial f(u, v)}{\partial v} \leq 0, \quad \frac{\partial g(u, v)}{\partial u} \leq 0$.

(H2)

$$
\begin{aligned}
& A_{i}=\left\{(u, v): \frac{\partial^{i} f(u, v)}{\partial v^{i}}=0, \quad(u, v) \in \mathbb{R}^{2}\right\}, \quad i=1,2, \cdots, 2 m, \\
& B_{j}=\left\{(u, v): \frac{\partial^{j} g(u, v)}{\partial u^{j}}=0, \quad(u, v) \in \mathbb{R}^{2}\right\}, \quad j=1,2, \cdots, 2 n .
\end{aligned}
$$

Assume

$$
\begin{aligned}
& A_{1} \subset A_{i}, \quad i=2,3, \cdots, 2 m, \quad B_{1} \subset B_{j}, \quad j=2,3, \cdots, 2 n ; \\
& \left.\frac{\partial^{2 m+1} f(u, v)}{\partial v^{2 m+1}}\right|_{A_{1}}<0,\left.\quad \frac{\partial^{2 n+1} g(u, v)}{\partial u^{2 n+1}}\right|_{B_{1}}<0 .
\end{aligned}
$$

(H4) For every bounded set $U$ in $\mathbb{R}$, the sets $\{f(u, v): u \in \mathbb{R}, v \in U\}$ and $\{g(u, v): u \in U, v \in \mathbb{R}\}$ are bounded.

Received by the editors September 14, 2005 and, in revised form, February 13, 2006. 2000 Mathematics Subject Classification. Primary 35L65; Secondary 74J30, 35L45.

Key words and phrases. Viscous conservation laws, travelling wave profile, a priori estimate. 
About these assumptions, condition (H1) implies that the system when $\epsilon=0$ is hyperbolic, that is, the matrix $\left(\nabla_{(u, v)} f(u, v), \nabla_{(u, v)} g(u, v)\right)$ has two real eigenvalues $\lambda_{1}(u, v) \leq \lambda_{2}(u, v)$ with corresponding right eigenvectors $r_{1}(u, v)$ and $r_{2}(u, v)$. In the following discussion, $r_{i}(u, v) \cdot \nabla_{(u, v)} \lambda_{i}, i=1,2$, may change sign so that the characteristic fields can be neither genuinely nonlinear nor linearly degenerate; cf. [5]. The conditions (H2) and (H3) are used to prove some monotone properties of the function $(u, v)$ along the travelling wave profile where some higher derivatives of the solution $(u, v)$ are considered. Basically, the condition (H2) ensures that the higher order derivatives $\frac{\partial^{i} f(u, v)}{\partial v^{i}}=0$ (or $\left.\frac{\partial^{i} g(u, v)}{\partial u^{i}}=0\right)$ up to the order $2 m$ (or $2 n$ ) at the points when $\frac{\partial f(u, v)}{\partial v}=0$ (or $\frac{\partial g(u, v)}{\partial u}=0$ ), while (H3) ensures that the next derivatives have a definite sign. Finally, the last condition (H4) is used to obtain the boundedness on the travelling wave solution.

In fact, for a given constant $s$ as the wave speed, a travelling wave profile $(u(\xi), v(\xi))$ with $\xi=x-s t$ to the system (1.1) satisfies the following differential equations:

$$
\left\{\begin{array}{l}
\varepsilon u^{\prime \prime}=f^{\prime}(u, v)-s u^{\prime}, \\
\varepsilon v^{\prime \prime}=g^{\prime}(u, v)-s v^{\prime},
\end{array}\right.
$$

where ' denotes differentiation with respect to $\xi$.

The main purpose of this paper is to show that for any given constant states $\left(u^{-}, v^{-}\right)$and $\left(u^{+}, v^{+}\right)$satisfying the Rankine-Hugoniot condition

$$
s=\frac{f\left(u^{+}, v^{+}\right)-f\left(u^{-}, v^{-}\right)}{u^{+}-u^{-}}=\frac{g\left(u^{+}, v^{+}\right)-g\left(u^{-}, v^{-}\right)}{v^{+}-v^{-}} \neq 0,
$$

there exists a solution to (1.2) with boundary condition

$$
(u(-\infty), v(-\infty))=\left(u^{-}, v^{-}\right), \quad(u(\infty), v(\infty))=\left(u^{+}, v^{+}\right) .
$$

This statement will be proved in the next section by obtaining the a priori estimate on the boundedness of the travelling wave solution profiles. Note also that the result in this paper does not include the case when $s=0$.

\section{A PRIORI ESTIMATE}

As in 1, 2, for the global existence of solutions to (1.2) and (1.4), it is sufficient to establish the a priori estimate. For this, we first consider the problem in a bounded domain with two parameters $\mu$ and $L$ as in the following theorem; cf. [1].

Theorem 2.1. Consider the boundary value problem

$$
\left\{\begin{array}{l}
\varepsilon u^{\prime \prime}=\mu f^{\prime}(u, v)-s u^{\prime}, \\
\varepsilon v^{\prime \prime}=\mu g^{\prime}(u, v)-s v^{\prime},
\end{array}\right.
$$

with

$$
(u(-L), v(-L))=\left(\mu u^{-}, \mu v^{-}\right), \quad(u(L), v(L))=\left(\mu u^{+}, \mu v^{+}\right) .
$$

Here the parameters $\mu$ and $L$ satisfy $\mu \in[0,1]$ and $L \geq 1$. Under the conditions (H1)-(H3), the solution $(u(\xi), v(\xi))$ of (2.1) and (2.2) has exactly one of the following properties:

1. $u(\xi)$ and $v(\xi)$ are constants on $[-L, L]$; 
2. $u(\xi)$ is increasing (or decreasing) with no critical points in $[-L, L]$, while $v(\xi)$ has at most one critical point in $(-L, L)$ corresponding to a local maximum (or minimum);

3. $v(\xi)$ is increasing (or decreasing) with no critical points in $[-L, L]$, while $u(\xi)$ has at most one critical point in $(-L, L)$ corresponding to a local maximum (or minimum).

Remark 2.1. Note that when $\mu=0$, the system (2.1) is a simple system of linear equations. Moreover, for finite $L>0$, the problem (2.1) and (2.2) can be solved by the standard contraction mapping theorem. Since the original problem (1.2) and (1.4) correspond to the case when $\mu=1$ and $L=\infty$, as in [1], it suffices to prove a uniform bound on the solutions to (2.1) and (2.2) which is independent of $\mu$ and $L$.

Proof of Theorem 2.1. Let $(u(\xi), v(\xi))$ be a non-constant solution of $(2.1)$ on $[-L, L]$. If $\xi_{0}$ is a critical point of $u(\xi)$. In the following, we only consider the case when

$$
\frac{\partial}{\partial v} f\left(u\left(\xi_{0}\right), v\left(\xi_{0}\right)\right)=0 .
$$

It is because the proof for the case when (2.3) does not hold is simpler, which corresponds to $m=0$ in the following discussion.

By using the condition (H2), (2.3) implies that

$$
\frac{\partial^{i}}{\partial v^{i}} f\left(u\left(\xi_{0}\right), v\left(\xi_{0}\right)\right)=0, \quad i=2,3, \cdots, 2 m
$$

(H3) gives

$$
\frac{\partial^{2 m+1}}{\partial v^{2 m+1}} f\left(u\left(\xi_{0}\right), v\left(\xi_{0}\right)\right)<0 .
$$

Differentiating the first equation in $(2.1)$ with respect to $\xi(k-2)$ times, for $3 \leq$ $k \leq 2 m+2$, we have the following expression for $u^{k}$ :

$$
\begin{aligned}
\varepsilon u^{(k)}= & \mu \sum_{i=1}^{k-2} F_{i}^{k}\left(u, v, u^{\prime}, v^{\prime}, \cdots, u^{(k-2)}, v^{(k-2)}\right) u^{(i)} \\
& +\mu \sum_{i=1}^{k-2} \frac{\partial^{i} f(u, v)}{\partial v^{i}} G_{i}^{k}\left(v^{\prime}, v^{\prime \prime}, \cdots, v^{(k-1)}\right) \\
& +\mu \frac{\partial f(u, v)}{\partial u} u^{(k-1)}+\mu \frac{\partial^{k-1} f(u, v)}{\partial v^{k-1}}\left(v^{\prime}\right)^{k-1}-s u^{(k-1)},
\end{aligned}
$$

where $F_{i}^{k}, G_{i}^{k}$ are smooth functions of their variables.

By (2.1), (2.3)-(2.6) and (H1)-(H3), we have

$$
u^{\prime}\left(\xi_{0}\right)=u^{\prime \prime}\left(\xi_{0}\right)=\cdots=u^{(2 m+1)}\left(\xi_{0}\right)=0 .
$$

Now by using (2.5) again, (2.6) and (2.7) imply that there are three possibilities of solution behavior at $\xi=\xi_{0}$ :

(a) $u^{(2 m+2)}\left(\xi_{0}\right)<0, v^{\prime}\left(\xi_{0}\right)>0$;

(b) $u^{(2 m+2)}\left(\xi_{0}\right)>0, v^{\prime}\left(\xi_{0}\right)<0$;

(c) $u^{(2 m+2)}\left(\xi_{0}\right)=0, v^{\prime}\left(\xi_{0}\right)=0$.

By the uniqueness of solution to (2.1) and (2.2) (cf. Lemma 4.1 in [1]) case (c) corresponds to $(u(\xi), v(\xi))$ being constant in $[-L, L]$. In other words, the critical 
point of $u(\xi)$ should be either a local minimum or a local maximum for the nonconstant function $u(\xi)$.

By symmetry, the same argument applies to the function $v(\xi)$. That is, if $\xi_{0}$ is a critical point of the non-constant function $v(\xi)$ and

$$
\frac{\partial}{\partial u} g\left(u\left(\xi_{0}\right), v\left(\xi_{0}\right)\right)=0,
$$

then either $v^{(2 n+2)}\left(\xi_{0}\right)<0, u^{\prime}\left(\xi_{0}\right)>0$ or $v^{(2 n+2)}\left(\xi_{0}\right)>0, u^{\prime}\left(\xi_{0}\right)<0$ for $n \geq 1$.

On the other hand, if $\xi_{0}$ is a critical point of the non-constant $v(\xi)$ and

$$
\frac{\partial}{\partial u} g\left(u\left(\xi_{0}\right), v\left(\xi_{0}\right)\right) \neq 0,
$$

then either $v^{\prime \prime}\left(\xi_{0}\right)<0, u^{\prime}\left(\xi_{0}\right)>0$ or $v^{\prime \prime}\left(\xi_{0}\right)>0, u^{\prime}\left(\xi_{0}\right)<0$.

Therefore, if $\xi_{0}$ is a critical point of the non-constant function $u(\xi)$ (or $v(\xi)$ ), then $\xi_{0}$ must be either a point of local maximum or minimum of $u(\xi)$ (or $v(\xi)$ ) with $v^{\prime}\left(\xi_{0}\right) \neq 0$ (or $u^{\prime}\left(\xi_{0}\right) \neq 0$ ).

We now turn to proving that non-constant function $u(\xi)$ has at most one critical point in $(-L, L)$ by contradiction. Assume that there are two different critical points, denoted by $\tau, \eta$ of $u(\xi)$ with $-L<\tau<\eta<L$. The above argument implies that $\tau$ is a local maximum (or minimum) and $\eta$ is a local minimum (or maximum) with $u^{\prime}(\xi)<0$ (or $u^{\prime}(\xi)>0$ ) for $\xi \in(\tau, \eta)$. Since we have $v^{\prime}(\tau)>0$ (or $v^{\prime}(\tau)<0$ ) and $v^{\prime}(\eta)<0$ (or $v^{\prime}(\eta)>0$ ); this implies that $v(\xi)$ attains a local maximum (or minimum) at some point $\xi_{1} \in(\tau, \eta)$. The above discussion again implies that $u^{\prime}\left(\xi_{1}\right)>0$ (or $u^{\prime}\left(\xi_{1}\right)<0$ ), which contradicts the property of $u(\xi)$ in the interval $(\tau, \eta)$. By symmetry, the same argument shows that the non-constant function $v(\xi)$ has at most one critical point in $(-L, L)$.

Finally, we will show that among non-constant functions $u(\xi)$ and $v(\xi)$, at most one of them has critical points in $(-L, L)$. Again, this can be proved by contradiction. Assume that $\tau$ is a critical point of $u(\xi)$ and $\eta$ is a critical point of $v(\xi)$. The above argument shows that $u(\xi)$ and $v(\xi)$ cannot attain local extremums at the same point. Without loss of generality, we can assume $\tau<\eta$. By the above discussion, if $\tau$ is a local maximum (or minimum) point of $u(\xi)$, then $v^{\prime}(\tau)>0$ (or $v^{\prime}(\tau)<0$ ). This in turn implies that $\eta$ must be a local maximum (or minimum) point of $v(\xi)$. However, in this case, we also have $u^{\prime}(\eta)>0$ (or $u^{\prime}(\eta)<0$ ) which implies that $\tau$ cannot be a local maximum (or minimum) point of $u(\xi)$. This contradiction implies that at most one of the non-constant functions $u(\xi)$ and $v(\xi)$ can have a critical point in $(-L, L)$. This completes the proof of the theorem.

The next theorem gives the a priori estimate on the boundedness of the solutions to $(2.1)$ and $(2.2)$.

Theorem 2.2 (A priori estimate). Let $(u(\xi), v(\xi))$ be a solution of (2.1) and (2.2). Then the solution has the following properties:

(i) There exists a constant $M$ depending only on $u^{-}, v^{-}, u^{+}, v^{+}, f$ and $g$ which is then independent of $L \geq 1$ and $\mu \in[0,1]$, such that

$$
\sup _{(-L, L)}|u(\xi)| \leq M, \quad \sup _{(-L, L)}|v(\xi)| \leq M .
$$


(ii) When $\mu=1$ and $L=\infty$, there exists a constant $N$ depending only on $u^{-}, v^{-}, u^{+}, v^{+}, f$ and $g$, such that

$$
\sup _{(-\infty, \infty)}|u(\xi)| \leq N, \quad \sup _{(-\infty, \infty)}|v(\xi)| \leq N .
$$

Proof. By Theorem 2.1, we know that at least one of $u(\xi)$ and $v(\xi)$ is monotone. If both $u(\xi)$ and $v(\xi)$ are monotone, the theorem obviously holds. Thus, in what follows, we assume only one of the functions $u(\xi)$ and $v(\xi)$ is monotone. Without loss of generality, we assume $v(\xi)$ is increasing. Then by Theorem 2.1, there exists a constant $\xi_{0} \in(-L, L)$ so that $u(\xi)$ is increasing on $\left(-L, \xi_{0}\right)$, while it is decreasing on $\left(\xi_{0}, L\right)$. Thus, the uniform bound on $v(\xi)$ is obvious, and we only need to consider $u(\xi)$ as follows. Since the wave speed $s$ is assumed to be non-zero, the discussion can be divided into the following two cases.

Case 1. When $s>0$, by integrating the first equation in $(2.1)$ over $\left(\xi_{0}, L\right)$, we have

$$
\varepsilon u^{\prime}(L)-\varepsilon u^{\prime}\left(\xi_{0}\right)=-s u^{+}+s u\left(\xi_{0}\right)+\mu f\left(\mu u^{+}, \mu v^{+}\right)-\mu f\left(u\left(\xi_{0}\right), v\left(\xi_{0}\right)\right) .
$$

Thus, $u^{\prime}\left(\xi_{0}\right)=0$ and $u^{\prime}(L) \leq 0$ yield

$$
\begin{aligned}
u\left(\xi_{0}\right) & =u^{+}+\frac{\mu}{s} f\left(u\left(\xi_{0}\right), v\left(\xi_{0}\right)\right)-\frac{\mu}{s} f\left(\mu u^{+}, \mu v^{+}\right)+\frac{\varepsilon}{s} u^{\prime}(L) \\
& \leq u^{+}+\frac{\mu}{s} f\left(u\left(\xi_{0}\right), v\left(\xi_{0}\right)\right)-\frac{\mu}{s} f\left(\mu u^{+}, \mu v^{+}\right) .
\end{aligned}
$$

By noting that $\left|v\left(\xi_{0}\right)\right|$ and $\mu v^{+}$are bounded by $\max \left\{\left|v^{-}\right|,\left|v^{+}\right|\right\}$, the assumption (H4) on $f$ yields that the right-hand side of (2.12) is bounded by a constant $I$ which is independent of $L \geq 1, \mu \in[0,1]$ and $\varepsilon>0$. Hence $|u(\xi)|$ is bounded by $\max \left\{\left|u_{-}\right|,\left|u_{+}\right|,|I|\right\}$.

Case 2. When $s<0$, by integrating the first equation in $(2.1)$ over $\left(-L, \xi_{0}\right)$, we have

$$
\varepsilon u^{\prime}\left(\xi_{0}\right)-\varepsilon u^{\prime}(-L)=-s u\left(\xi_{0}\right)+s u^{-}+\mu f\left(u\left(\xi_{0}\right), v\left(\xi_{0}\right)\right)-\mu f\left(\mu u^{-}, \mu v^{-}\right) .
$$

Hence, $u^{\prime}\left(\xi_{0}\right)=0$ and $u^{\prime}(-L) \geq 0$ give

$$
\begin{aligned}
u\left(\xi_{0}\right) & =u^{-}+\frac{\mu}{s} f\left(u\left(\xi_{0}\right), v\left(\xi_{0}\right)\right)-\frac{\mu}{s} f\left(\mu u^{-}, \mu v^{-}\right)+\frac{\varepsilon}{s} u^{\prime}(-L) \\
& \leq u^{-}+\frac{\mu}{s} f\left(u\left(\xi_{0}\right), v\left(\xi_{0}\right)\right)-\frac{\mu}{s} f\left(\mu u^{-}, \mu v^{-}\right) .
\end{aligned}
$$

Similar to Case 1, (2.14) and (H4) imply that $u(\xi)$ is bounded independent of $\mu$ and $L$.

Therefore, the proof of the theorem is complete.

Similar to the argument in 1, 2, the uniform boundedness of solutions to the problem (2.1) and (2.2) immediately gives the existence of travelling wave profiles stated in the following theorem.

Theorem 2.3. Under the assumptions (H1)-(H4), (1.2) and (1.4) admits a solution $(u(\xi), v(\xi))$ when the speed of wave $s \neq 0$.

Remark 2.2. When the assumptions (H1) and (H3) are replaced by the following ones denoted by (H5) and (H6):

(H5) $\quad \frac{\partial f(u, v)}{\partial v} \geq 0, \quad \frac{\partial g(u, v)}{\partial u} \geq 0$ 


$$
\left.\frac{\partial^{2 m+1} f(u, v)}{\partial v^{2 m+1}}\right|_{A_{1}}>0,\left.\quad \frac{\partial^{2 n+1} g(u, v)}{\partial u^{2 n+1}}\right|_{B_{1}}>0 \text {, the existence of travelling }
$$

wave solutions to (1.2) and (1.4) with non-zero wave speed still holds.

Example. Here we use the following system to illustrate Theorem 2.3 on the existence of travelling wave profiles. Consider

$$
\left\{\begin{array}{l}
u_{t}-\frac{1}{2 m+1}\left(v^{2 m+1}\right)_{x}=\varepsilon u_{x x} \\
v_{t}-\left(u+\frac{1}{3} A u^{3}\right)_{x}=\varepsilon v_{x x}
\end{array}\right.
$$

where $m \geq 1$ is an integer and $A \geq 0$ is a constant. Note that the above system when $\epsilon=0$ and $A=0$ was studied in 3 , 4.

A travelling wave with speed $s \neq 0$ to the system (2.15) satisfies the differential equations

$$
\left\{\begin{array}{l}
\varepsilon u^{\prime \prime}=-s u^{\prime}-\frac{1}{2 m+1}\left(v^{2 m+1}\right)^{\prime}, \\
\varepsilon v^{\prime \prime}=-s v^{\prime}-\left(u+\frac{1}{3} A u^{3}\right)^{\prime},
\end{array}\right.
$$

with boundary condition

$$
(u(-\infty), v(-\infty))=\left(u^{-}, v^{-}\right), \quad(u(\infty), v(\infty))=\left(u^{+}, v^{+}\right) .
$$

Note that the two eigenvalues of (2.15) are

$$
\lambda_{\mp}=\mp\left(1+A u^{2}\right)^{\frac{1}{2}} v^{m},
$$

with corresponding right eigenvectors given by

$$
r_{\mp}=\left(v^{m}, \pm\left(1+A u^{2}\right)^{\frac{1}{2}}\right)^{T} .
$$

Thus,

$$
\nabla \lambda_{\mp} \cdot r_{\mp}=\mp v^{m-1}\left(1+A u^{2}\right)^{-\frac{1}{2}}\left(A u v^{m+1} \pm m\left(1+A u^{2}\right)^{\frac{3}{2}}\right) .
$$

Since $\lambda_{-}=\lambda_{+}$when $v=0$, the system is not strictly hyperbolic. Moreover, the first (or second) characteristic field is linearly degenerate (cf. [5]) when

$$
\begin{gathered}
v^{m-1}\left(A u v^{m+1}+m\left(1+A u^{2}\right)^{\frac{3}{2}}\right)=0 \\
\left(\text { or } \quad v^{m-1}\left(A u v^{m+1}-m\left(1+A u^{2}\right)^{\frac{3}{2}}\right)=0\right) .
\end{gathered}
$$

Thus, when $A>0$ and $m \geq 2$, the characteristic fields of the system (2.15) are neither genuinely nonlinear nor linearly degenerate. However, it is straightforward to check that the assumptions (H1)-(H4) hold so that the system (2.15) admits a travelling wave solution $(u(\xi), v(\xi))$ for any given end states at $\pm \infty$ in $(2.17)$.

\section{ACKNOWLEDGMENTS}

First, the authors would like to thank the referee for useful comments on the revision of this paper. Second, the research of the first author was supported by Hong Kong RGC Competitive Earmarked Research Grant, CityU103004. The research of the second author was supported by the Program for New Century Excellent Talents of Universities \#NCET-04-0745 and the Key Project of National Natural Science Foundation of China \#10431060. 


\section{REFERENCES}

[1] Dafermos C.M., Solution of the Riemann problem for a class of hyperbolic systems of conservation laws by the viscosity method, Arch. Rational Mech. Anal., 52(1973), 1-9. MR0340837 $(49: 5587)$

[2] Dafermos C.M., DiPerna R.J., The Riemann problem for certain classes of hyperbolic systems of conservation laws, J. Differential Equations, 20(1976), 90-114. MR0404871 (53:8671)

[3] Keyfitz B.L., Kranzer H.C., A system of nonstrictly hyperbolic conservation laws arising in elasticity theory, Arch. Rational Mech. Anal., 72(1980), 219-241. MR0549642 (80k:35050)

[4] Keyfitz B.L., Kranzer H.C., The Riemann Problem for a class of hyperbolic conservation laws exhibiting a parabolic degeneracy, J. Differential Equations, 47(1983),35-65. MR0684449 (84a:35162)

[5] Lax P.D., Hyperbolic systems of conservation laws II, Comm. Pure Appl. Math., 10(1957), 537-556. MR0093653 (20:176)

[6] Liu T.P., The Riemann problem for general $2 \times 2$ conservation laws, Trans. Amer. Math. Soc., 199(1974), 89-112. MR0367472 (51:3714)

[7] Schaeffer D.G., Shearer M., Riemann problems for nonstrictly hyperbolic $2 \times 2$ systems of conservation laws, Trans. Amer. Math. Soc., 304(1987), 267-305. MR0906816 (88m:35101)

[8] Slemrod M., Tzavaras A.E., A limiting viscosity approach for the Riemann problem in isentropic gas dynamics, Indiana Univ. Math. J., 38(1989), 1047-1073. MR.1029688 (90m:35119)

[9] Smoller J.A., On the solution of the Riemann problem with general step data for an extended class of hyperbolic systems, Mich. Math. J., 16(1969), 201-210. MR0247283 (40:552)

[10] Smoller J.A., Johnson J.L., Global solutions for an extended class of hyperbolic systems of conservation laws, Arch. Rational Mech. Anal., 32(1969), 168-189. MR0236527 (38:4822)

Department of Mathematics, City University of Hong Kong, Tat Chee Avenue, KowloOn, Hong Kong

E-mail address: matyang@cityu.edu.hk

Department of Mathematics, City University of Hong Kong, Tat Chee Avenue, KOWLOON, HONG KONG

E-mail address: meizhang3@student.cityu.edu.hk

Laboratory of Nonlinear Analysis, Department of Mathematics, Central China Normal University, Wuhan 430079, People's Republic of China

E-mail address: cjzhu@mail.ccnu.edu.cn 